\title{
Effects on glycocalyx structures of frozen-thawed bovine sperm induced by flow cytometry and artificial capacitation
}

\author{
Kohei UMEZU ${ }^{1)}$, Yuuki HIRADATE'), Takashi NUMABE'), Kenshiro HARA ${ }^{1)}$ and \\ Kentaro TANEMURA ${ }^{1)}$ \\ 1)Laboratory of Animal Reproduction and Development, Graduate School of Agricultural Science, Tohoku University, \\ Miyagi 980-0845, Japan \\ ${ }^{2)}$ Miyagi Agricultural Development Corporation, Miyagi 981-0914, Japan
}

\begin{abstract}
Sperm sorting by flow cytometry is a useful technology in the bovine industry, but the conception rates after artificial insemination using sex-sorted sperm are lower than when using the un-sorted sperm. In this study, we have investigated the causes for these low conception rates. We have focused on changes caused by flow cytometry to the glycocalyx, which forms the outermost surface of the sperm membrane. We have also evaluated the effects of capacitation on the glycocalyx since capacitation involves a redistribution of the sperm membrane that is vital for successful fertilization and conception. Lectin histochemistry was used to visualize the structure of the sperm glycocalyx. Lectin-staining sites were examined in non-treated sperm, sex-sorted sperm, and capacitated sperm. We have detected six different staining patterns related to different labeling regions of the sperm. Phaseolus vulgaris-erythroagglutinin (PHA-E) lectin-staining patterns of non-treated sperm were very different from those observed for sex-sorted sperm or capacitated sperm, suggesting that both, sex sorting by flow cytometry and the capacitation process affected the glycocalyx structures in the sperm. In addition, the total tyrosine-phosphorylation level in sex-sorted sperm was significantly higher than that in the non-treated sperm. Therefore, we concluded that the unexpected capacitation of bovine sperm during flow cytometry is associated with changes in the glycocalyx. Since premature capacitation leads to low conception rates, this unexpected capacitation could be a cause of low conception rates after artificial insemination using sex-sorted sperm.
\end{abstract}

Key words: Bovine sperm, Capacitation, Flow cytometry, Glycocalyx, Lectin

(J. Reprod. Dev. 63: 473-480, 2017)

$\mathbf{S}^{\mathrm{p}}$ perm sorting that permits sex preselection is a useful technology in the bovine industry because sex-specific artificial insemination promises substantial benefits for cattle breeding and production. For example, sperm sexing would allow the specific production of male calves for beef production and female calves for milk production or cattle replacement. Pre-selection of spermatozoa by flow cytometry based on DNA differences between X-and Y-chromosome-bearing populations is an established method that has been introduced into commercial cattle production. Some researchers have documented the ability of flow cytometry to consistently produce an approximately $90 \%$ sex bias among cattle offspring $[1,2]$. This technology has been successfully applied to accurate sorting of sperm in more than 23 mammalian species, and normal offspring have been produced via artificial insemination or in vitro fertilization with sex-sorted sperm in at least seven species [3, 4].

However, conception rates after artificial insemination using bovine sex-sorted semen are lower than when using conventional semen [5]. Several studies reported that the flow-cytometric procedure had

Received: May 8, 2017

Accepted: June 19, 2017

Published online in J-STAGE: July 13, 2017

C2017 by the Society for Reproduction and Development

Correspondence: K Tanemura (e-mail: kentaro@m.tohoku.ac.jp)

This is an open-access article distributed under the terms of the Creative Commons Attribution Non-Commercial No Derivatives (by-nc-nd) License. (CC-BY-NC-ND 4.0: https://creativecommons.org/licenses/by-nc-nd/4.0/) detrimental effects on sperm, possibly leading to low conception rates [6-11]. Thus, it is necessary to clarify the effects of flow cytometry on bovine sperm. Sperm is subjected to physical stresses in the flow cytometer, including high-pressures and laser exposure, suggesting that the cell surface could be particularly affected by flow cytometry.

The cell surface of all living cells is enveloped in a glycocalyx consisting of many glycoconjugates. The glycocalyx is involve in many biological events in vivo, including cell adhesion, cell signaling, immune responses, and differentiation [12, 13]. The sperm surface is also enveloped in the glycocalyx that is thicker than that of typical somatic cells [14]. As touched upon previously, the glycocalyx that constitutes the outermost surface of the sperm membrane could be damaged by flow cytometry.

The glycocalyx is critically important for sperm survival in the female reproductive tract. It allows the sperm to penetrate the cervical mucus; it protects sperm from humoral and cellular immunity in the uterus, where sperm encounter female antibodies; and it inhibits premature capacitation in areas of the female reproductive tract that are not the site of fertilization [14-18]. The structure of the sperm glycocalyx is very dynamic [19] and reflects the characteristics of both the sperm and the extracellular environment $[18,20]$. Thus, it is important to understand the organization of the sperm glycocalyx in more detail because it is an observable characteristic that potentially could be used to evaluate sperm quality.

Lectin staining has been widely used to visualize cell-surface structures because of their ability to recognize specific components of 
the glycocalyx. The bovine sperm glycocalyx has been investigated using lectins in several studies. For example, ConA, PNA and PSA lectins bind to the acrosome regions of bovine sperm and have been used to evaluate the acrosome reaction $[21,22]$. It is also known that sites binding with WGA lectin in bovine sperm are altered during sperm capacitation [23]. Together, these observations suggest that sperm functions such as the acrosome reaction and sperm capacitation are related to the structures of the glycocalyx. In particular, sperm capacitation involves a redistribution of glycoconjugates on the sperm surface that is vital for the successful fertilization $[18,24]$. It appears that premature capacitation of sperm is one of the causes of low conception rates, and that the sperm glycocalyx is able to inhibit the premature capacitation $[18,25,26]$. Therefore, evaluating the effects of capacitation on the structures of sperm glycocalyx may help us to elucidate the relationship between these structures and the success of fertilization or conception.

The aim of this study was to determine the effects of flow cytometry and sperm capacitation on the glycocalyx of bovine sperm, and to identify causes of low conception rates after artificial insemination using sex-sorted sperm. We used lectin histochemistry to visualize the structure of the sperm glycocalyx and investigated lectin-staining sites in non-treated sperm, in sex-sorted sperm separated by flow cytometry, and in artificially capacitated sperm.

\section{Materials and Methods}

\section{Sperm preparation}

Three kinds of frozen bovine semen were obtained from ZEN-NOH LIVESTOC; non-treated sperm (Japanese Black cattle, from three different bulls); and X-sperm (Holstein) and Y-sperm (Japanese Black cattle) separated by flow cytometry [27]. Sperm were thawed in a water bath at $38^{\circ} \mathrm{C}$ for $15 \mathrm{sec}$, placed in $1.5-\mathrm{ml}$ capped plastic tubes, and $1000 \mu \mathrm{l}$ of phosphate-buffered saline (PBS, pH 7.4) was added. After centrifugation at $2000 \mathrm{rpm}$ at room temperature for 5 min, the supernatant was discarded; the sperm were then resuspended in $1000 \mu \mathrm{l}$ of PBS and centrifuged again. The supernatant was again discarded and the sperm were resuspended in PBS at a concentration of $5 \times 10^{7}$ cells $/ \mathrm{ml}$. Samples of each sperm were smeared onto glass slides and dried in air.

\section{Artificial capacitation and Western blotting}

Sperm capacitation was induced using the heparin assay described previously [28, 29]. Briefly, non-treated sperm (Japanese Black cattle, from three different bulls) were incubated in BGM-1 (bovine gamete medium 1) added to $10 \mu \mathrm{g} / \mathrm{ml}$ heparin for $4 \mathrm{~h}$ at $38^{\circ} \mathrm{C}$ under $5 \%$ $\mathrm{CO}_{2}$ in air. Sperm were then washed with PBS, centrifuged twice, resuspended in PBS at a concentration of $5 \times 10^{7}$ cells $/ \mathrm{ml}$, smeared onto glass slides, and dried in air, as described above.

Sperm capacitation was evaluated by Western blot analysis of tyrosine phosphorylation, which is the major indicator of sperm capacitation $[30,31]$. Non-treated sperm, capacitated sperm and sexsorted sperm (Y-sperm) before the smear procedure were centrifuged at $10,000 \mathrm{rpm}$ at $4^{\circ} \mathrm{C}$ for $5 \mathrm{~min}$, and the sperm pellets were collected. The sperm pellets were washed with PBS and centrifuged again at $10,000 \mathrm{rpm}$. After the supernatants were discarded, Sample Buffer Solution (Nacalai Tesque, Kyoto, Japan) was added to extract the proteins. The obtained proteins were separated by $12 \%$ SDS-PAGE and transferred to polyvinylidene membranes. After 60 min of blocking with $1 \%$ bovine serum albumin in tris-buffered saline containing $0.1 \%$ Tween ${ }^{\circledR} 20$ (TBS-T, pH 7.5-7.8), the membrane was treated overnight at $4{ }^{\circ} \mathrm{C}$ with a mouse monoclonal anti-phosphotyrosine $4 \mathrm{G} 10$ antibody (05-321, 1:20000; Merck Millipore, Darmstadt, Germany) or with anti- $\alpha$-tubulin (T9026, 1:10000; Merck Millipore) as an internal control. After three washes with TBS-T, the membrane was treated with horseradish peroxidase (HRP)-conjugated anti-mouse immunoglobulin G antibody (1:2000; Promega, Madison, Wisconsin, USA) for $2 \mathrm{~h}$ at room temperature. Detection was performed with Chemilumi One (Nacalai Tesque) and images were obtained using an LAS-3000-mini Lumino Image Analyzer (Fujifilm, Tokyo, Japan). Densitometric analyses were performed using Image Gauge software v4.22 (Fujifilm).

\section{Lectin histochemistry}

Seventeen biotinylated lectins with different specificities were used (J Oil Mills, Tokyo, Japan) (Table 1). Sperm smears on glass slides were blocked with Blocking One (Nacalai Tesque) in a humid chamber for $1 \mathrm{~h}$. After blocking, $4 \mu \mathrm{g} / \mathrm{ml}$ of each lectin was added to the slide in a humid chamber for $1.5 \mathrm{~h}$ at room temperature. The smears were rinsed with PBS three times for $3 \mathrm{~min}$, and stained with $1 \mu \mathrm{g} / \mathrm{ml}$ of Streptavidin Alexa Fluor 488 (Thermo Fisher Scientific, Waltham, Massachusetts, USA) and $0.5 \mu \mathrm{g} / \mathrm{ml}$ of Hoechst 33342 (Thermo Fisher Scientific). After rinsing with PBS, the smears were covered with coverslips and examined under a confocal laser-scanning microscope (LSM-710, Carl Zeiss, Jena, Germany). We defined six staining patterns in the lectin-stained region and used them to classify each sperm sample. Ten fields were randomly photographed in each semen slide, and the proportions of each of the staining patterns were calculated. Counting of over 200 sperm was performed for each lectin and each semen (non-treated sperm, capacitated sperm and sex-sorted sperm).

\section{Statistical analysis}

All experiments were replicated at least three times. Data are presented as means \pm the standard error (SE). Statistical analyses were carried out using analysis of variance (ANOVA) and Fisher's protected least significant difference test. Values of $\mathrm{P}<0.05$ were considered to indicate significant differences $(* * \mathrm{P}<0.01, * \mathrm{P}<0.05)$.

\section{Results}

In this study, three kinds of frozen bovine semen (non-treated sperm, capacitated sperm and sex-sorted sperm) were stained with 17 different lectins. Lectin labeling in the sperm head was observed with 15 of the lectins, excluding DBA and UEA I. Among these 15 lectins, the sperm exhibited distinctly different labeling regions with the same lectin; i.e., the labeling patterns on the bovine sperm head by lectin histochemistry were not constant. Thus, we detected six staining patterns among the regions labeled by lectins, and classified each sperm field on the basis of these patterns (Fig. 1). The six patterns were defined as follows: pattern 1 (P1), highly stained acrosomal region; pattern $2(\mathrm{P} 2)$, highly stained in the equatorial segment; pattern 3 (P3), no labeling; pattern 4 (P4), faint labeling 
Table 1. Taxonomic names and main specificities of lectins used in this study

\begin{tabular}{lll}
\hline \multicolumn{1}{c}{ Lectin } & Abbreviation & Major sugar recognized \\
\hline Concanavalin A & ConA & Man $\alpha$, Glc $\alpha$ \\
Datura stramonium Lectin & DSL & Gal $\beta 1-4$ GlcNAc \\
Dolichos biflorus Agglutinin & DBA & GalNAc $\alpha$ \\
Griffonia simplicifolia Lectin I & GSL I & Gal $\alpha$, GalNAc $\alpha$ \\
Griffonia simplicifolia Lectin II & GSL II & GlcNAc $\alpha$, GlcNAc $\beta$ \\
Lectin isolated from Artocarpus integrifolia Seeds & Jacalin & Gal $\beta 3$ GalNAc \\
Lens culinaris (Lentil) Agglutinin & LCA & Man $\alpha$ \\
Lycopersicon esculentum (Tomato) Lectin & LEL & (GlcNAc) $n$ \\
Phaseolus vulgaris Erythroagglutinin & PHA-E & Gal $\beta(1,4)$ GlcNAc \\
Peanut Agglutinin & PNA & Gal $\beta 1-3$ GalNAc \\
Pisum sativum Agglutinin & PSA & Man $\alpha$ \\
Ricinus communis Agglutinin I & RCA120 & Gal, GalNAc \\
Soybean Agglutinin & SBA & GalNAc \\
Ulex europaeus Agglutinin I & UEA I & Fuc $\alpha$ \\
Vicia villosa Lectin & VVL & GalNAc \\
Wheat Germ Agglutinin & WGA & (GlcNAc) n, sialic acid \\
Succinylated Wheat Germ Agglutinin & s-WGA & (GlcNAc) $n$ \\
\hline
\end{tabular}

Gal: D-Galactose, GalNAc: N-Acetyl-D-galactosamine, Glc: D-Glucose, GlcNAc: N-Acetyl-D-glucosamine, Fuc: L-Fucose, Man: D-Mannose.

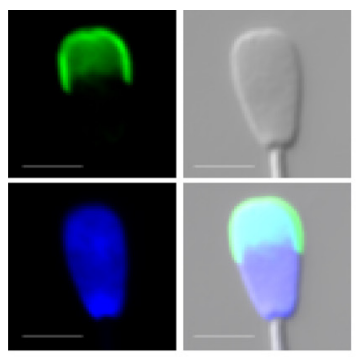

Pattern 1

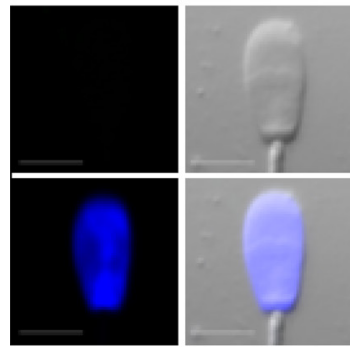

Pattern 3

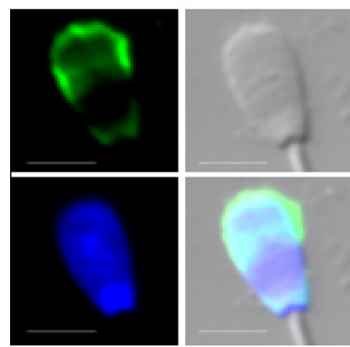

Pattern 5
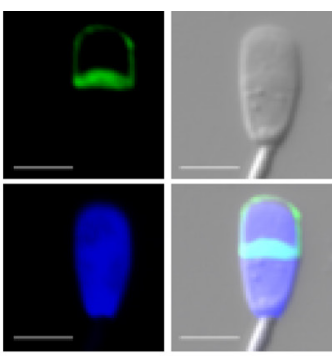

Pattern 2

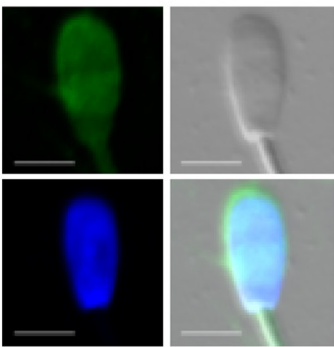

Pattern 4

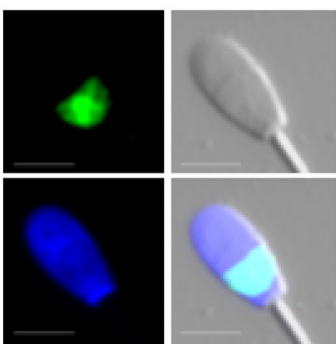

Pattern 6 all over the head; pattern 5 (P5), acrosomal and post-acrosomal region stained with no labeling in the equatorial segment; pattern 6 (P6), highly stained post-acrosomal region. Figure 2 indicates the staining patterns observed with each of the lectins. At least three different staining patterns were observed with each of the 15 lectins; thus, the lectin-staining patterns in frozen-thawed bovine sperm were quite variable.

Next, we calculated the relative proportions of each lectin-staining pattern exhibited by each of the 15 lectins using the above classification of six patterns (Fig. 3). The proportions observed in non-treated sperm, capacitated sperm and sex-sorted sperm were compared to evaluate the effects of flow cytometry and artificial capacitation on the lectin-staining patterns of bovine sperm.

In non-treated sperm and sex-sorted sperm, P1 was the most common staining pattern for most lectins (Fig. 3A-C). In contrast, in capacitated sperm, the proportion of pattern P3 was higher than that of non-treated sperm and sex-sorted sperm (Fig. 3D). In particular, WGA and s-WGA showed the highest proportions of $\mathrm{P} 3$ in capacitated sperm ( $42.9 \%$ and $46.8 \%$, respectively). In these lectins, the proportion of P3 of artificially capacitated sperm was significantly higher than that of non-treated sperm and sex-sorted sperm. That is, artificial capacitation increased the ratio of $\mathrm{P} 3$ in bovine sperm.

With Phaseolus vulgaris-erythroagglutinin (PHA-E), P5 was the most represented lectin-staining patterns in non-treated sperm (Fig. 3A), whereas P1, P2 and P3 dominated in capacitated sperm, and

Fig. 1. Models of the lectin-staining patterns. Six patterns were defined: pattern 1 , highly stained acrosomal region; pattern 2 , highly stained in the equatorial segment; pattern 3, no labeling; pattern 4, faint labeling all over the head; pattern 5 , acrosomal and post-acrosomal region stained with no labeling in the equatorial segment; pattern 6, highly stained post-acrosomal region. Blue: nuclear stain (Hoechst 33342); green: lectin-staining sites. Bars $=5 \mu \mathrm{m}$. 


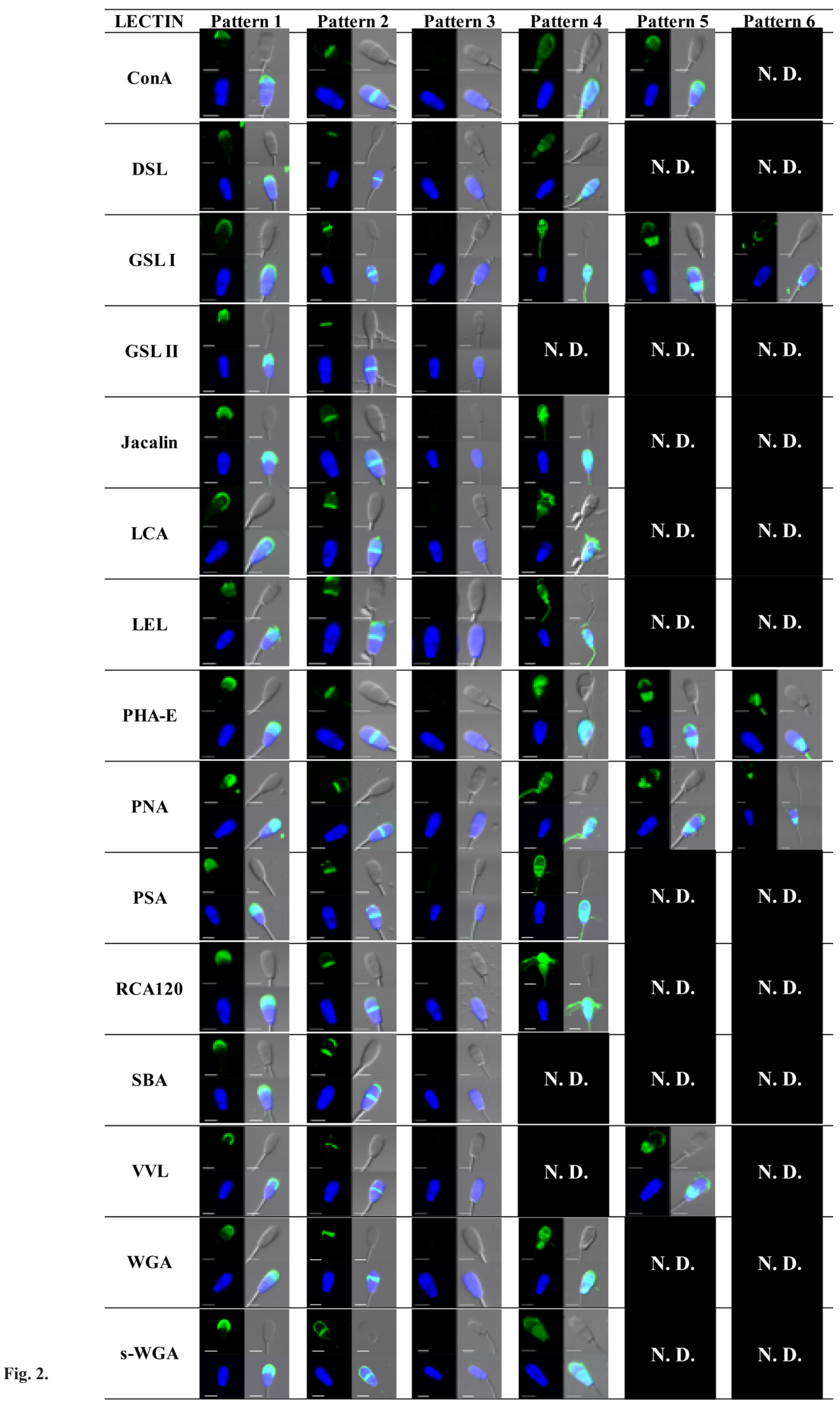




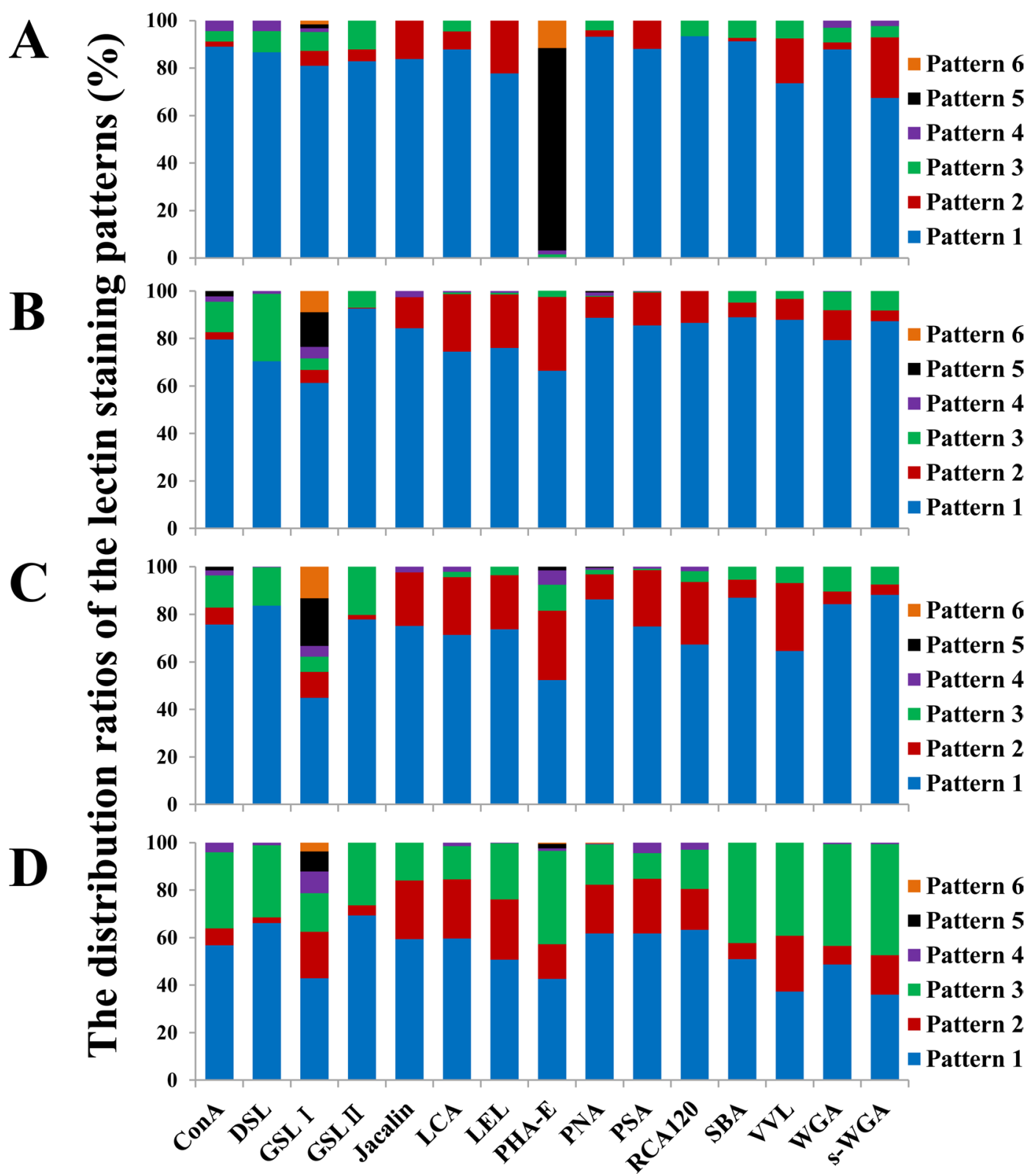

Fig. 3. Distribution proportions of the lectin-staining patterns in non-treated sperm, sex-sorted sperm (X-sperm and Y-sperm) and capacitated sperm. Ten fields of sperm of were randomly photographed and the proportions of each staining pattern were calculated for each lectin. In non-treated sperm (A) and sex-sorted sperm (B: X-sperm; C: Y-sperm), most lectins exhibited staining pattern P1. In contrast, in capacitated sperm (D), the P3 was in higher proportion relative to non-treated sperm and sex-sorted sperm. With PHA-E, P5 was the main lectin-staining patterns in non-treated sperm, whereas P1, P2 and P3 were dominant in capacitated sperm, and P1 and P2 were dominant in sex-sorted sperm.

Fig. 2. Sperm distribution based on lectin-staining patterns with each lectin. Non-treated sperm, sex-sorted sperm or capacitated sperm were stained with biotinylated lectins. The staining patterns with each lectin were observed under a confocal laser scanning microscope and sperm were grouped into six staining patterns. More than three staining patterns were observed with the all 15 lectins that labeled sperm head in this study. Blue: nuclear (Hoechst 33342), Green: lectin-staining sites, N. D.: not detected. Bars $=5 \mu \mathrm{m}$.

P1 and P2 dominated in sex-sorted sperm (Fig. 3B-D). The PHA-E staining pattern of non-treated sperm was significantly different from that in capacitated sperm, non-treated sperm, and sex-sorted sperm. Thus, flow cytometry and artificial capacitation exert a significant effect on PHA-E staining patterns in bovine sperm. Furthermore, no marked differences were observed between X-sorted sperm and Y-sorted sperm for any of the lectins used in this study.

Finally, western blotting of sperm-protein tyrosine phosphorylation, which is a major indicator of sperm capacitation, was performed 
to elucidate the relationship between the effects of flow cytometry and sperm capacitation. The total tyrosine-phosphorylation levels of sex-sorted sperm and artificially capacitated sperm were significantly higher than that of non-treated sperm, and no significant differences between sex-sorted sperm and artificially capacitated sperm were observed (Fig. 4).

\section{Discussion}

In this study, bovine sperm were stained with 17 different lectins to accurately characterize the structure of the sperm glycocalyx. Based on lectin labeling of different regions of the sperm, we defined six discrete staining patterns (P1-P6). Several different staining patterns have also been observed by lectin histochemistry in human and mouse sperm [20,32]. These results suggest that lectin-staining patterns and glycocalyx structures in bovine and other mammalian sperm are quite varied.

In non-treated sperm and sex-sorted sperm, P1 was the most common lectin-staining pattern with most lectins (Fig. 3A-C). This observation indicates that the glycocalyx in bovine sperm head is concentrated in the acrosome region. RCA120, PNA, SBA, ConA and PSA showed the highest proportions of $\mathrm{P} 1$ in non-treated sperm (93.4, 93.2, 91.3, 89.0 and 88.1\%, respectively). Previous studies reported that ConA, PNA and PSA bind specifically to the acrosome region of bovine sperm $[21,22]$. It has been reported that the sperm acrosome may be damaged by smearing onto a glass slide, so that the glycocalyx in the acrosome region may have been damaged in a few sperm in our experiments [33]. Possibly, sperm with intact acrosomes showed pattern P1 and a few with damaged acrosomes showed other patterns with ConA, PNA and PSA labeling. Therefore, our data also suggest that ConA, PNA and PSA bind specifically to the acrosome region of bovine sperm. Although an association between the acrosome and RCA 120 with specificity toward terminal Galß1-4GlcNAc in glycocalyx has not been reported, it was reported that SBA, which has specificity toward terminal GalNAc in the glycocalyx, binds specifically to the acrosome region of rat and hamster sperm [34-36]. In addition, SBA was strongly reactive in the acrosome region of the elongating bovine spermatids [37]. These studies together with our own observations suggest that SBA binds specifically to the acrosome region of bovine ejaculated sperm. Therefore, SBA could be a new marker to determine the acrosome status of bovine sperm, in addition to ConA, PNA and PSA.

In capacitated sperm, the proportion of $\mathrm{P} 3$ was higher than that of non-treated sperm and sex-sorted sperm (Fig. 3D). This suggests that artificial capacitation induced loss of lectin binding to some bovine sperm heads. In particular, WGA and s-WGA showed the highest proportions of P3 in capacitated sperm (42.9 and 46.8\%, respectively). WGA has a specificity toward GlcNAc and sialic acids, and s-WGA has a specificity toward $\beta$-GlcNAc $[38,39]$. Thus, we suggest that GlcNAc and sialic acids on the bovine sperm head were especially affected by artificial capacitation. Likewise, several studies have reported that lectin-staining sites on the sperm head in several species were changed during capacitation $[23,32$, 40]. Therefore, capacitation is an event during which the structure of the sperm glycocalyx is dynamically changed.

Next, we will discuss the reason why sperm capacitation has such
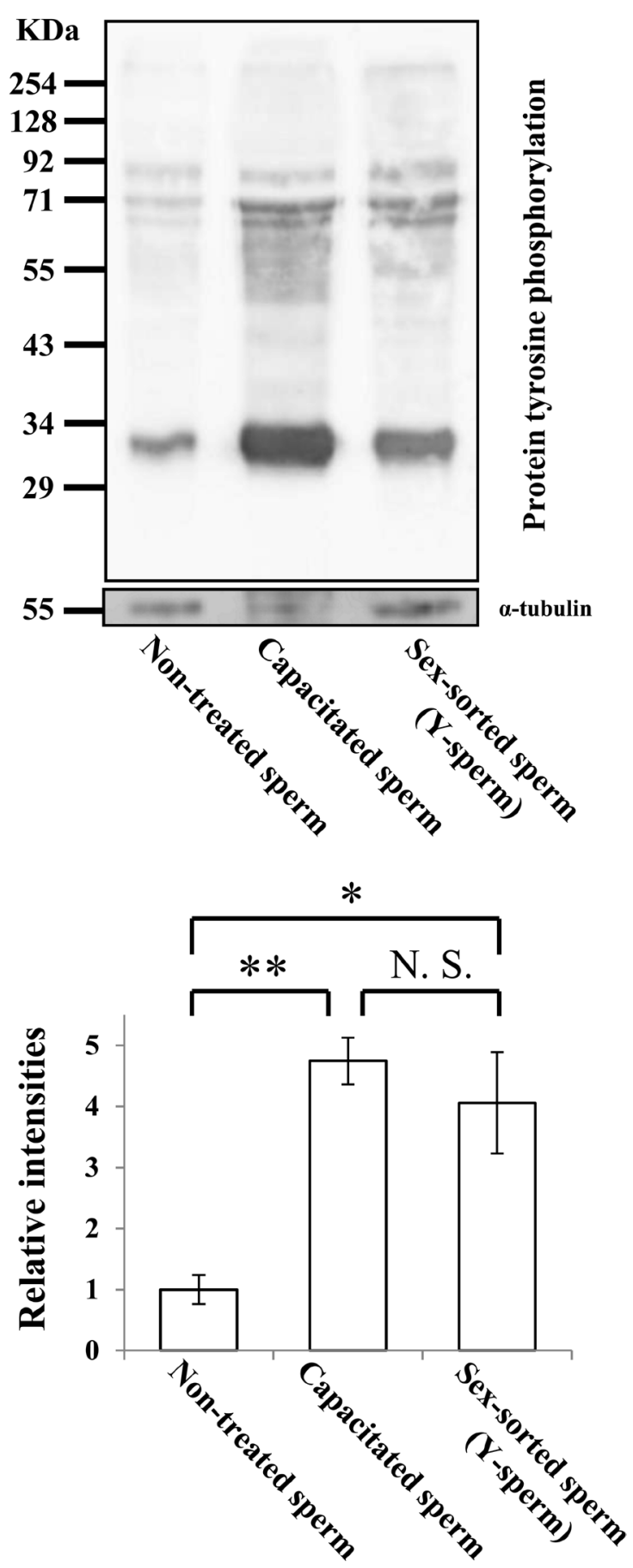

Fig. 4. Western blot analysis of protein tyrosine phosphorylation. Protein tyrosine phosphorylation in non-treated sperm, capacitated sperm and sex-sorted sperm (Y-sperm) was revealed by Western blotting. The upper panel shows representative blotting images for at least three repeated experiments. Note that the total tyrosine phosphorylation levels of capacitated sperm and sexsorted sperm are significantly higher than that of non-treated sperm. The lower panel shows quantification and comparison of the total quantities of tyrosine phosphorylated proteins among non-treated sperm, capacitated sperm, and sex-sorted sperm. The protein bands of non-treated sperm were assigned a base value of 1. $\alpha$-Tubulin was used as a loading control. Data are shown as means \pm SE. $(* \mathrm{P}<0.05, * * \mathrm{P}<0.01, \mathrm{~N}$. S.: not significant $)$ 
effects on sperm. It is known that sperm membranes undergo a number changes during capacitation, including removal of glycoproteins and cholesterol [24]. Presumably, these changes in the sperm membrane are a consequence of changes in the structure of the glycocalyx that constitutes the outer surface of the sperm membrane. Thus, one possible explanation is that capacitation causes the changes in the structure of the sperm membrane, including the glycocalyx, which induce changes in the lectin-staining sites in the sperm head. In particular, sialic acids on the sperm head are cleaved by sperm sialidase during capacitation, so that it is considered that the WGAstaining sites are especially affected by capacitation [41]. However, because the s-WGA staining sites were most affected by capacitation in this study, it is necessary to examine the interaction between capacitation and $\beta$-GlcNAc on the sperm head. That may contribute to the elucidation of further details of the mechanism of fertilization and to the application of s-WGA as a capacitation marker in bovine sperm. Another possible explanation is that sperm capacitation causes a spontaneous acrosome reaction, which may result in a loss of lectin binding to the acrosome region of bovine sperm.

The PHA-E staining patterns of non-treated sperm were quite different from those in sex-sorted sperm, non-treated sperm and capacitated sperm. This suggests that flow cytometry and artificial capacitation exert a significant effect on PHA-E-staining patterns in bovine sperm. PHA-E has a specificity toward bisecting GlcNAc with two Gal residues on each arm, and this structure on the post-acrosomal region of the bovine sperm head may be particularly affected by flow cytometry and artificial capacitation [42]. The reason why this structure is especially affected by flow cytometry is unknown and further studies are required for the elucidation of the functions of this glycocalyx in sperm.

The effects of both flow cytometry and capacitation on PHAE-staining patterns were that P1, P2 and P3 mainly replaced P5. Therefore, it seems that both processes had similar effects on bovine sperm. In addition, Western blotting showed that the total tyrosine phosphorylation level of sex-sorted sperm was significantly higher than that of non-treated sperm. Because total tyrosine phosphorylation is a major indicator of sperm capacitation, this observation suggests that the sex-sorted sperm was closer to capacitation status than non-treated sperm was. Several studies have reported that flow cytometry induced membrane destabilization of sperm, resembling the membrane status during capacitation $[9,10]$. These observations imply that flow cytometry induced unexpected capacitation, which affected the structure of glycocalyx, in some of the bovine sperm. Possibly, PHA-E could be used to evaluate damage caused to bovine sperm by flow cytometry, when this procedure is used commercially in bovine reproduction.

Currently, the low conception rates after artificial insemination using sex-sorted sperm have become a problem in bovine reproduction, but the causes of this problem are not well known [5]. The present study is the first conclusive description of the effects of flow cytometry on the structure of the glycocalyx of bovine sperm. We suggest that these effects induce unexpected capacitation. Because premature capacitation of sperm is one of the causes of low conception rates [25], we infer that unexpected capacitation, as observed in this study, could be a cause of low conception rates after artificial insemination using sex-sorted sperm. The sperm glycocalyx also mediates numerous functions in the female reproductive tract, including passage through the cervical mucus, protection from female immunity, and masking sperm proteins involved in fertilization [18, 19, 26, 43-45]. Therefore, because the sperm glycocalyx is important for sperm survival in the female reproduction tract and for the success of fertilization, it is also possible that the changes on the structure of glycocalyx observed in this study may have detrimental effects on these functions, contributing to the low conception rates after artificial insemination using sex-sorted sperm. This study has identified a possible new cause of low conception rates after artificial insemination using sex-sorted sperm. Further studies will be required to clarify the interaction between the structure of the glycocalyx and conception rates after artificial insemination in cattle.

This study was the first attempt to compare lectin-staining patterns of X-sorted and Y-sorted sperm, although no marked differences were noted. However, more rigorous investigations are required because the X-sorted sperm was derived from a Holstein bull, while the Y-sorted sperm was derived from Japanese black cattle in this study.

In summary, the diversity of lectin-staining patterns observed in this study could be caused by sperm capacitation, the acrosome reaction, damage to the sperm membrane during the smear procedure, or by flow cytometry; the effect was similar in X-sperm and Y-sperm. Although the relationship between the diversity of lectin-staining patterns and the condition of individual sperm is unclear, it is possible that lectin-staining patterns could provide information concerning the quality of individual sperm. These individual differences could provide the female with a means to select particular sperm.

Overall, this study has revealed the diversity of glycocalyx structure in bovine sperm and the effects of flow cytometry and capacitation on glycocalyx structure. In the industrial framework of bovine reproduction, artificial insemination using frozen bovine semen is generally employed, and sex-sorted semen has also been used commercially. The next step should be to clarify the interaction between glycocalyx structures in bovine sperm and conception rates after artificial insemination. This may lead to more efficient reproduction in cattle and the development of lectins as new sperm markers. Finally, the many functions of the sperm glycocalyx have only begun to be appreciated; future studies of this membrane component will provide a greater understanding of its structure and function in sperm and of the molecular mechanisms of fertilization.

\section{Acknowledgments}

The authors wish to thank the Miyagi Livestock Experimental Station for the gift of bovine spermatozoa. This study was supported by the Meat Inspection Office, Sendai, Japan.

\section{References}

1. Seidel GE Jr, Schenk JL, Herickhoff LA, Doyle SP, Brink Z, Green RD, Cran DG. Insemination of heifers with sexed sperm. Theriogenology 1999; 52: 1407-1420. [Medline] [CrossRef]

2. Schenk JL, Suh TK, Cran DG, Seidel GE Jr. Cryopreservation of flow-sorted bovine spermatozoa. Theriogenology 1999; 52: 1375-1391. [Medline] [CrossRef]

3. Garner DL. Flow cytometric sexing of mammalian sperm. Theriogenology 2006; 65 943-957. [Medline] [CrossRef]

4. Garner DL, Seidel GE Jr. History of commercializing sexed semen for cattle. Theriogenology 2008; 69: 886-895. [Medline] [CrossRef] 
5. Dejarnette JM, Leach MA, Nebel RL, Marshall CE, McCleary CR, Moreno JF. Effects of sex-sorting and sperm dosage on conception rates of Holstein heifers: is comparable fertility of sex-sorted and conventional semen plausible? J Dairy Sci 2011; 94: 3477-3483. [Medline] [CrossRef]

6. Garner DL. Sex-Sorting mammalian sperm: concept to application in animals. J Androl 2001; 22: 519-526. [Medline]

7. Rath D, Johnson LA. Application and commercialization of flow cytometrically sexsorted semen. Reprod Domest Anim 2008; 43(Suppl 2): 338-346. [Medline] [CrossRef]

8. Rath D, Moench-Tegeder G, Taylor U, Johnson LA. Improved quality of sex-sorted sperm: a prerequisite for wider commercial application. Theriogenology 2009; 71: 22-29. [Medline] [CrossRef]

9. Bucci D, Galeati G, Tamanini C, Vallorani C, Rodriguez-Gil JE, Spinaci M. Effect of sex sorting on CTC staining, actin cytoskeleton and tyrosine phosphorylation in bull and boar spermatozoa. Theriogenology 2012; 77: 1206-1216. [Medline] [CrossRef]

10. Balao da Silva CM, Ortega Ferrusola C, Morillo Rodriguez A, Gallardo Bolaños JM, Plaza Dávila M, Morrell JM, Rodriguez Martínez H, Tapia JA, Aparicio IM, Peña FJ. Sex sorting increases the permeability of the membrane of stallion spermatozoa. Anim Reprod Sci 2013; 138: 241-251. [Medline] [CrossRef]

11. Quan GB, Ma Y, Li J, Wu GQ, Li DJ, Ni YN, Lv CR, Zhu L, Hong QH. Effects of Hoechst33342 staining on the viability and flow cytometric sex-sorting of frozen-thawed ram sperm. Cryobiology 2015; 70: 23-31. [Medline] [CrossRef]

12. Gupta G, Surolia A, Sampathkumar SG. Lectin microarrays for glycomic analysis. OMICS 2010; 14: 419-436. [Medline] [CrossRef]

13. Maverakis E, Kim K, Shimoda M, Gershwin ME, Patel F, Wilken R, Raychaudhuri S, Ruhaak LR, Lebrilla CB. Glycans in the immune system and The Altered Glycan Theory of Autoimmunity: a critical review. J Autoimmun 2015; 57: 1-13. [Medline] [CrossRef]

14. Schröter S, Osterhoff C, McArdle W, Ivell R. The glycocalyx of the sperm surface. Hum Reprod Update 1999; 5: 302-313. [Medline] [CrossRef]

15. Pandya IJ, Cohen $\mathbf{J}$. The leukocytic reaction of the human uterine cervix to spermatozoa. Fertil Steril 1985; 43: 417-421. [Medline] [CrossRef]

16. Thompson LA, Barratt CL, Bolton AE, Cooke ID. The leukocytic reaction of the human uterine cervix. Am J Reprod Immunol 1992; 28: 85-89. [Medline] [CrossRef]

17. Tollner TL, Yudin AI, Treece CA, Overstreet JW, Cherr GN. Macaque sperm coating protein DEFB126 facilitates sperm penetration of cervical mucus. Hum Reprod 2008; 23: 2523-2534. [Medline] [CrossRef]

18. Tecle E, Gagneux P. Sugar-coated sperm: Unraveling the functions of the mammalian sperm glycocalyx. Mol Reprod Dev 2015; 82: 635-650. [Medline] [CrossRef]

19. Diekman AB. Glycoconjugates in sperm function and gamete interactions: how much sugar does it take to sweet-talk the egg? Cell Mol Life Sci 2003; 60: 298-308. [Medline] [CrossRef]

20. Gómez-Torres MJ, Avilés M, Girela JL, Murcia V, Fernández-Colom PJ, Romeu A, De Juan J. Characterization of the lectin binding pattern in human spermatozoa after swim-up selection. Histol Histopathol 2012; 27: 1621-1628. [Medline]

21. Cross NL, Watson SK. Assessing acrosomal status of bovine sperm using fluoresceinated lectins. Theriogenology 1994; 42: 89-98. [Medline] [CrossRef]

22. Nishikimi A, Yamada M, Minami N, Utsumi K. Evaluation of acrosomal status of bovine spermatozoa using concanavalin a lectin. Theriogenology 1997; 48: 1007-1016. [Medline] [CrossRef]

23. Medeiros CM, Parrish JJ. Changes in lectin binding to bovine sperm during heparininduced capacitation. Mol Reprod Dev 1996; 44: 525-532. [Medline] [CrossRef]

24. Gadella BM, Tsai PS, Boerke A, Brewis IA. Sperm head membrane reorganisation during capacitation. Int J Dev Biol 2008; 52: 473-480. [Medline] [CrossRef]

25. Kuroda K, Fukushima M, Harayama H. Premature capacitation of frozen-thawed spermatozoa from subfertile Japanese black cattle. J Reprod Dev 2007; 53: 1079-1086. [Medline] [CrossRef]

26. Yeung WS, Lee KF, Koistinen R, Koistinen H, Seppala M, Ho PC, Chiu PC. Glycodelin: a molecule with multi-functions on spermatozoa. Soc Reprod Fertil Suppl 2007; 63:
143-151. [Medline]

27. Seidel GE Jr. Overview of sexing sperm. Theriogenology 2007; 68: 443-446. [Medline] [CrossRef]

28. Parrish JJ, Susko-Parrish J, Winer MA, First NL. Capacitation of bovine sperm by heparin. Biol Reprod 1988; 38: 1171-1180. [Medline] [CrossRef]

29. Umezu K, Hiradate Y, Oikawa T, Ishiguro H, Numabe T, Hara K, Tanemura K. Exogenous neurotensin modulates sperm function in Japanese Black cattle. J Reprod Dev 2016; 62: 409-414. [Medline] [CrossRef]

30. Galantino-Homer HL, Visconti PE, Kopf GS. Regulation of protein tyrosine phosphorylation during bovine sperm capacitation by a cyclic adenosine 35 -monophosphatedependent pathway. Biol Reprod 1997; 56: 707-719. [Medline] [CrossRef]

31. Visconti PE, Kopf GS. Regulation of protein phosphorylation during sperm capacitation. Biol Reprod 1998; 59: 1-6. [Medline] [CrossRef]

32. Baker SS, Thomas M, Thaler CD. Sperm membrane dynamics assessed by changes in lectin fluorescence before and after capacitation. J Androl 2004; 25: 744-751. [Medline] [CrossRef]

33. Almadaly E, El-Kon I, Heleil B, Fattouh S, Mukoujima K, Ueda T, Hoshino Y, Takasu M, Murase T. Methodological factors affecting the results of staining frozen-thawed fertile and subfertile Japanese Black bull spermatozoa for acrosomal status. Anim Reprod Sci 2012; 136: 23-32. [Medline] [CrossRef]

34. Piller V, Piller F, Cartron JP. Comparison of the carbohydrate-binding specificities of seven N-acetyl-D-galactosamine-recognizing lectins. Eur J Biochem 1990; 191: 461-466. [Medline] [CrossRef]

35. Ballesta J, Martínez-Menárguez JA, Pastor LM, Avilés M, Madrid JF, Castells MT. Lectin binding pattern in the testes of several tetrapode vertebrates. Eur J Basic Appl Histochem 1991; 35: 107-117. [Medline]

36. Martínez-Menárguez JA, Ballesta J, Avilés M, Castells MT, Madrid JF. Cytochemica characterization of glycoproteins in the developing acrosome of rats. An ultrastructural study using lectin histochemistry, enzymes and chemical deglycosylation. Histochemistry 1992; 97: 439-449. [Medline] [CrossRef]

37. Arya M, Vanha-Perttula T. Lectin-binding pattern of bull testis and epididymis. J Androl 1985; 6: 230-242. [Medline] [CrossRef]

38. Wakitani S, Hondo E, Shimokawa T, Kusakabe K, Okada T, Nakamuta N, Stewart CL, Kiso Y. Effects of leukemia inhibitory factor on lectin-binding patterns in the uterine stromal vessels of mice. Immunobiology 2008; 213: 143-150. [Medline] [CrossRef]

39. Saito S, Taniguchi K. Expression patterns of glycoconjugates in the three distinctive olfactory pathways of the clawed frog, Xenopus laevis. J Vet Med Sci 2000; 62: 153-159. [Medline] [CrossRef]

40. Taitzoglou IA, Kokoli AN, Killian GJ. Modifications of surface carbohydrates on bovine spermatozoa mediated by oviductal fluid: a flow cytometric study using lectins. Int $J$ Androl 2007; 30: 108-114. [Medline] [CrossRef]

41. Ma F, Wu D, Deng L, Secrest P, Zhao J, Varki N, Lindheim S, Gagneux P. Sialidase on mammalian sperm mediate deciduous sialylation during capacitation. $J$ Biol Chem 2012; 287: 38073-38079. [Medline] [CrossRef]

42. Nagae M, Soga K, Morita-Matsumoto K, Hanashima S, Ikeda A, Yamamoto K, Yamaguchi Y. Phytohemagglutinin from Phaseolus vulgaris (PHA-E) displays a novel glycan recognition mode using a common legume lectin fold. Glycobiology 2014; 24 368-378. [Medline] [CrossRef]

43. Gilks CB, Reid PE, Clement PB, Owen DA. Histochemical changes in cervical mucussecreting epithelium during the normal menstrual cycle. Fertil Steril 1989; 51: 286-291. [Medline] [CrossRef]

44. Toshimori K, Araki S, Oura C, Eddy EM. Loss of sperm surface sialic acid induces phagocytosis: an assay with a monoclonal antibody $\mathrm{T} 21$, which recognizes a $54 \mathrm{~K}$ sialoglycoprotein. Arch Androl 1991; 27: 79-86. [Medline] [CrossRef]

45. Varki A. Since there are PAMPs and DAMPs, there must be SAMPs? Glycan selfassociated molecular patterns dampen innate immunity, but pathogens can mimic them. Glycobiology 2011; 21: 1121-1124. [Medline] [CrossRef] 